\title{
Caspase expression profile and functional activity in a panel of breast cancer cell lines
}

\author{
SHIHE YANG ${ }^{1}$, JOAN LIU ${ }^{1}$, ANN D. THOR ${ }^{2}$ and XIAOHE YANG ${ }^{1}$ \\ ${ }^{1}$ Department of Pathology, University of Oklahoma Health Sciences Center, Oklahoma City, OK 73104; \\ ${ }^{2}$ Department of Pathology, University of Colorado Health Sciences Center, Aurora, CO 80045, USA
}

Received December 7, 2006; Accepted January 15, 2007

\begin{abstract}
Caspases play an essential role in the initiation/ regulation of apoptosis. Aberrant apoptotic regulation has been associated with carcinogenesis and therapeutic resistance. To explore the possible involvement of altered caspase expression in breast cancer, we have systematically examined the expression of both protein and mRNA levels of 7 caspases in a panel of 18 breast cancer cell lines. We found that variation of caspase expression can occur at both protein and RNA levels. Down-regulation of these caspases, especially caspase- 8 and -10 , was frequently observed. Functional screening of these selected cell lines using TNF- $\alpha$, doxorubicin and radiation induced cell injury showed that a lack of functional caspase- 8 resulted in resistance to TNF- $\alpha$-induced apoptosis. Array style examination of caspase expression profiles in breast cancer cell lines yields massive information that is valuable in establishing cell line models to study the role of caspase down-regulation/deficiency in breast cancer development and therapeutic resistance.
\end{abstract}

\section{Introduction}

Apoptosis, or programmed cell death, is an important mechanism that facilitates the cyclic proliferation and involution processes associated with the menstrual cycle, pregnancy and lactation (1). Intact apoptotic regulation is also required for auto-execution of cells in response to anti-cancer therapies. Abnormal apoptotic cascades and specific regulators have been reported in breast cancers. Defective apoptosis is believed to be responsible (at least in part) for breast carcinogenesis. Mechanistically, this process can be altered via critical tumor suppressor genes, such as p53, whose encoded protein induces apoptosis in response to DNA damage and other stresses. The

Correspondence to: Dr Xiaohe Yang, Department of Pathology, University of Oklahoma Health Sciences Center, Oklahoma City, OK 73104, USA

E-mail: xiaohe-yang@ouhsc.edu

Key words: caspase, breast cancer, transcription, chemotherapy, radiation p53 gene is mutated in approximately one third of breast cancers (2). Signaling proteins such as Bax, a proapoptotic factor of the Bcl-2 family, have also been shown to be altered in the majority of breast cancers $(3,4)$. p53 and Bax alterations have been associated with therapeutic resistance and significantly worse survival $(3,4)$. Defects in a myriad of other apoptotic regulators, such as the caspase enzymes, are also likely contributors to breast carcinogenesis and therapeutic resistance.

Numerous stimuli may induce apoptosis through the death receptor or mitochondrial pathways. These converge into a common pathway, known as the 'caspase cascade' (5). Caspases are a group of cysteine proteases that play a central role in both apoptotic initiation and execution because of their ability to activate proteins via a complex system of direct and feedback loops (6). To date, 14 mammalian caspases have been cloned. These have been subdivided into three major groups based on functional similarities: i) initiator or apical caspases, such as caspase-2, $-8,-9$ and -10; ii) effector caspases, such as caspase-3, -6 and -7 ; and iii) caspases involved in cytokine maturation and inflammation, such as caspase-1, - 4 and $-5(6,7)$. The regulation of caspases is believed to be primarily at the protein level. In the absence of an apoptotic signal, caspase proteins are inactive zymogens, or procaspases. Proapoptotic signals activate caspases by cleavage of these proteins, resulting in functional enzymes (8). Activation of the caspase cascade eventually results in proteolysis of numerous subcellular substrates, such as poly-(ADP-ribose) polymerase (PARP), and Lamin B. Cleavage of these critical proteins disables cellular processes and breaks down the subcellular cytoskeleton, resulting in the classical morphologic features of apoptosis such as nuclear fragmentation and blebbing (9).

Alterations of caspase expression appear to be a factor contributing to aberrant apoptosis in cancer cells. We and others have reported that caspase- 3 is deficient in MCF-7 breast cancer cells $(10,11)$. Its reconstitution significantly sensitizes these cells to doxorubicin, etoposide and radiationinduced apoptosis (11-13). Studies of human breast cancers indicate this may be an important and frequent phenomenon, as it accounts for approximately a $75 \%$ lack of caspase- 3 transcription and protein expression (14). Caspase-8 deficiency has been associated with both lung cancers and neuroblastomas $(15,16)$.

We hypothesize that caspase deficiency/down-regulation may be common in breast cancer, likely enhances cell survival, 
even in the setting of significant cell aberrations, thus promoting breast carcinogenesis and therapeutic resistance. To test this hypothesis, we performed a systematic examination of the expression profile of both protein and mRNA of multiple caspases, including caspase- $2,-3,-6,-7,-8,-9$ and -10 , in 18 breast cancer cell lines. We also performed functional screening of these cell lines for their response to doxorubicin, radiation or TNF- $\alpha$. These stimuli induce apoptosis through mitochondrial or death receptor pathways. Our results reveal that the caspase deficiency/down-regulation is very common in breast cancer cell lines and, when present, is associated with apoptotic resistance. Our data supports a role for caspases in breast cancer development and their frequent therapeutic resistance. The data may also be useful for investigators who work with these model systems, as marked heterogeneity exists.

\section{Materials and methods}

Reagents. Chemicals including 5-diphenyltetrazolium bromide (MTT), cycloheximide (CHX), DMEM/F12 medium and others were purchased from Sigma (St. Louis, MO). Antibodies against caspase-3, -6, -7, -10 and actin were purchased from Santa Cruz Biotechnology (Santa Cruz, CA). Antibodies against caspase-2, -8 or -9 were purchased from BD Biosciences (San Diego, CA). Horseradish peroxidase (HRP)labeled secondary antibodies were purchased from Amersham/ GE Health Care (Piscataway, NJ). Caspase multiplex PCR set-5 and 6 were purchased from BioSources (Carmarillo, CA).

Cell lines and cell culture. All the breast cancer cell lines used in this study, including MDA-MB-330, MDA-MB-483, HS-578T, MDA-MB-361, MDA-MB-468, MDA-MB-436, MDA-MB-415, BT-474, MDA-MB-415, MDA-MB-157, T47D, MCF-10A, ZR-75-1, MDA-MB-134VI, BT-20, SKBR-3, MDA-MB-453, MDA-MB-231, ZR-75-30, MDA-MB435s and MCF-10A were purchased from ATCC (Manassas, $\mathrm{VA})$. The cells were cultured in DMEM/F12 medium containing $10 \%$ fetal bovine serum (FBS).

Western blotting. Drug-treated or non-synchronized cells were lysed with a lysis buffer containing $50 \mathrm{mM}$ Tris, $5 \mathrm{mM}$ EDTA, $150 \mathrm{mM} \mathrm{NaCl}, 0.5 \% \mathrm{NP}-40$ and protease inhibitors. The protein concentration was determined using a BCA assay kit (Pierce Biotechnology Inc. Rockford, IL). Fifty micrograms of protein lysate was loaded onto each lane of a gel. Proteins were separated with 12 or $15 \%$ SDS-PAGE and transferred to a nitrocellulose membrane. The membrane was probed with the relevant, specific primary antibodies at a dilution of 1:3002000 , followed by washing and probing with an appropriate secondary antibody. Specific protein bands were visualized by autoradiography using an ECL kit (Amersham/GE Health Care).

TNF- $\alpha$ treatment and DAPI (4', 6-diamidino-2-phenylindole, dihydrochloride) staining. Cells from each line were inoculated into a $60-\mathrm{mm}$ dish at $1 \times 10^{6} /$ dish $24 \mathrm{~h}$ before treatment. The cells were then treated with $80 \mathrm{ng} / \mathrm{ml} \mathrm{TNF}-\alpha$ plus $5 \mu \mathrm{g} /$ $\mathrm{ml} \mathrm{CHX}$ for $4 \mathrm{~h}$, followed by trypsinization. Treated cells were fixed with $2 \%$ paraformaldehyde at $4^{\circ} \mathrm{C}$ for $30 \mathrm{~min}$. The cells were stained with $0.5 \mu \mathrm{g} / \mathrm{ml}$ DAPI for $30 \mathrm{~min}$. Stained cells were then washed and mounted on slides using a cytospinner. Nuclear morphology of the cells was visualized using an Olympus fluorescence microscope. Cells with nuclear condensation and/or nuclear fragmentation were counted as apoptotic cells (11). At least 300 cells were counted from each slide/sample. The apoptotic index (\% of cells in apoptosis) was calculated using the ratio of cells in apoptosis, divided by the total number of cells multiplied by 100 . The experiments were performed in triplicate.

MTT assay. MTT assays were used to evaluate cell death responses to various doses of doxorubicin and radiation. For doxorubicin treatment, the cells were inoculated into 96-well plates at $1 \times 10^{3}$ cells/well $24 \mathrm{~h}$ before treatment. The cells were then treated with increasing concentrations of doxorubicin ranging from 0.15 to $9000 \mathrm{nM}$, for 6 days. After removal of the medium, $50 \mu \mathrm{l}$ of MTT solution $(500 \mu \mathrm{g} / \mathrm{ml})$ was added to each well followed by 4-h incubation. MTT solution was replaced by DMSO to dissolve blue formazan crystals, and absorbance was measured at $562 \mathrm{~nm}$ using a microplate reader. For the radiation experiments, cells were inoculated into a $60-\mathrm{mm}$ dish at $1 \times 10^{6} / \mathrm{dish} 24 \mathrm{~h}$ before radiation. The cells were then irradiated at doses ranging from 0.5 to $32 \mathrm{~Gy}$ (at 3 time intervals) using a Varian Clinac 4 Photon Linear Accelerator and inoculated into 96-well plates at $1 \times 10^{3}$ cells/ well. MTT assay was performed 6 days after radiation. For $\mathrm{ID}_{50}$ determination from the MTT assay, non-linear regression analysis was performed with Cricket Graph software to generate curves for $\mathrm{ID}_{50}$ calculation.

RNA extraction, reverse transcription (RT) and multiplex PCR. Total-RNA was isolated from non-synchronized cell lines using RNeasy Mini Kit (Qiagen, Valencia, CA). First-strand cDNA synthesis was performed using SuperscriptIII ${ }^{\mathrm{TM}}$ First Strand synthesis system (Invitrogen, Carlsbad, CA). After reverse transcription, the cDNA samples derived from each cell line were used as templates. mRNA levels of specific caspases were detected using 2 sets of multiplex PCR kits, sets 5 and 6 in the apoptosis series of multiplex PCR kits from BioSource. Set 5 detects caspase-3, -5, -8, -9, Apoptosis Protease activating factor-1 (Apaf-1) and GAPDH. Set 6 detects caspase-2, -4, -6, -7, -10 and GAPDH. Multiplex PCR was performed following the manufacturer's protocols. Twenty microliters of PCR products were electrophoresed on $2 \%$ agarose gels and visualized by ethidium bromide staining. The images were acquired and analyzed using the UPVII gel documentation system. The relative mRNA levels of each caspase in different cell lines were semiquantitatively analyzed by normalization of the density of each specific band with GAPDH signals of the corresponding cell lines. Primers in set 5 multiplex were designed to detect caspase-2, -4, -6, -7, and -10 . Set 6 multiplex detected caspase-3, -5, -8, -9 and Apaf-1. Both sets used GAPDH as a housekeeping gene control.

\section{Results}

Protein levels of caspase-2, -3, -6, -7, -8, -9 and -10 in 18 breast cancer cell lines. We first examined the protein levels 


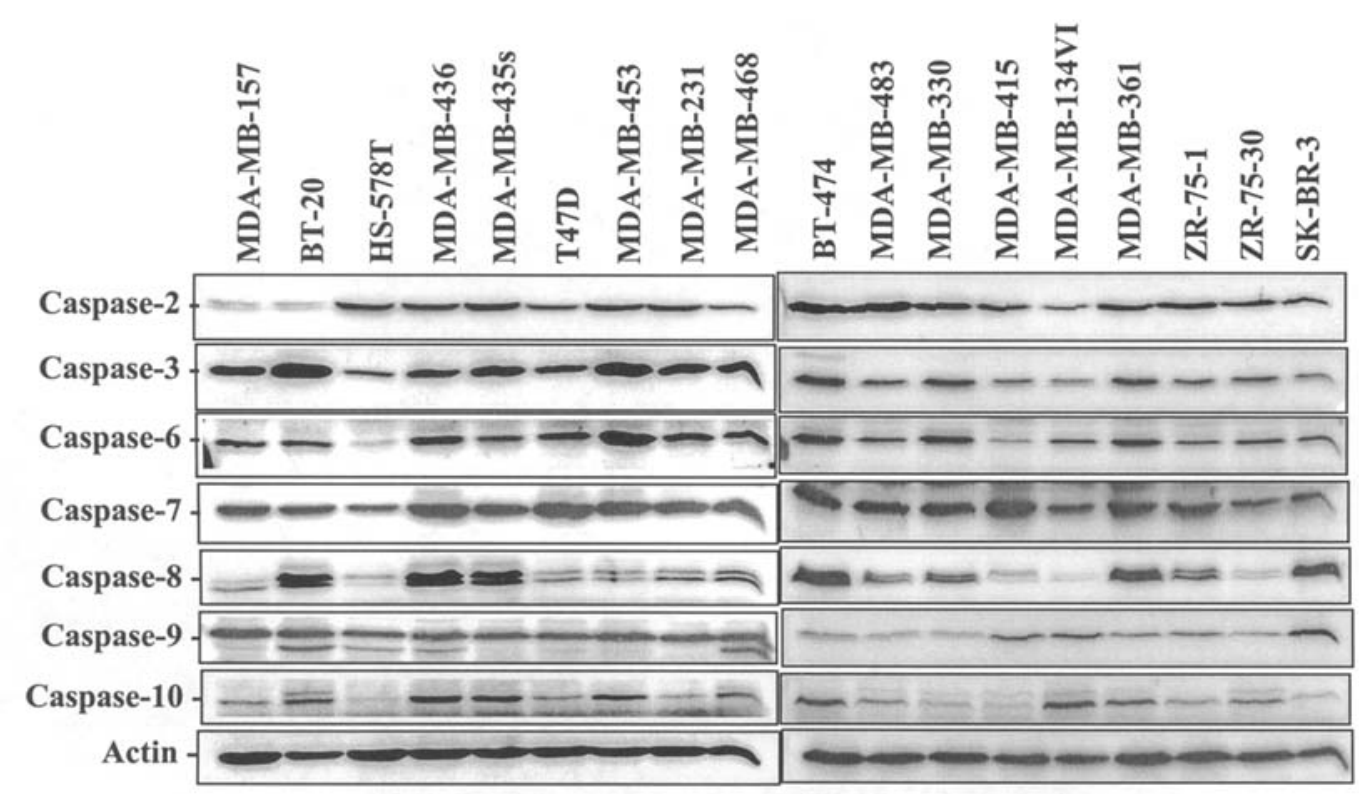

Figure 1. Protein levels of caspase-2, $-3,-6,-7,-8,-9$ and -10 in 18 breast cancer cell lines. Protein lysates of each cell line were made from non-synchronized cells. Individual caspases and actin were probed with corresponding specific antibodies using Western blotting.

of caspase- $2,-3,-6,-7,-8,-9$ and -10 in 18 breast cancer cell lines by Western blotting. Protein levels of each caspase varied significantly amongst different cell lines (Fig. 1). The caspase-2 level was abundant in most cell lines. It was very low only in two lines, MDA-MB-157 and BT-20 cells. It was somewhat low in three additional lines, T47D, MDA-MB-468 and MDA-MB-134VI cells. Caspase-3 was detectably expressed at low levels in five cell lines, including HS-578T, MDAMB-483, MDA-MB-415, MDA-MB-134VI and SK-BR-3 cells. BT-20 and MDA-MB-453 cells expressed the highest levels of caspase-3. Caspase-6 was expressed at very low levels in HS-578T, MDA-MB-435s, MDA-MB-415 and ZR75-1 cells. Caspase-7 was reduced in HS-578T, MDA-MB134VI and SK-BR-3 cells. Caspase- 8 levels were very low in MDA-MB-157, HS-578T, MDA-MB-134VI, ZR-75-30 and T47D cells. In contrast, BT-20, MDA-MB-435s, MDA-MB436, BT474 and MDA-MB-361 cells expressed high levels of caspase-8. ZR-75-30 and MDA-MB-330 expressed relatively low levels of caspase-9. For caspase-10, protein levels were lower in HS-578T, MDA-MB-415, SK-BR-3, MDA-MB-330, T47D, and MDA-MB231 cells. In aggregate, reduced levels of all caspases were generally common amongst the cell lines detected. In particular, variations in the levels of caspase- 8 and 10 were more prevalent than the other caspases. Fluctuation of caspase-7 and -9 was relatively less frequent.

Relative mRNA levels of caspase-2, -3, -4, -6, -7, -8, -9 and -10 and Apaf-1 in breast cancer cell lines. To examine whether caspase protein expression reflected mRNA levels, we extracted total-RNA from 18 breast cancer cell lines and a benign breast epithelial cell line (MCF-10A) and examined the mRNA levels of caspase-2, -3, -4, -6, -7, -8, -9 and -10, as well as Apaf-1 by multiplex PCR. As shown in Fig. 2, the mRNA levels of the caspases also varied significantly from cell line to cell line. Using an image analysis system, the relative levels of each mRNA band were normalized with GAPDH signals (data not shown). Caspase-2 mRNA levels were lowest in
ZR-75-30, SK-BR-3 and MDA-MB-231 cells. Caspase-3 mRNA was low in MDA-MB-415 cells as compared to other lines. Caspase-4 mRNA levels were not detectable in MDA-MB-134VI cells and were relatively lower in T47D and ZR-75-30 cells. Caspase-6 mRNA levels were lower in MDA-MB-134VI, ZR-75-1, MDA-MB-361 and BT-474 cells as compared to all other cell lines. Variations in caspase-7 mRNA between cell lines were not evident. Caspase- 8 mRNA levels were lower in HS-578T, MDA-MB-415, MDA-MB134VI and ZR-75-30 cells. Variation of caspase-10 mRNA levels was the most notable in the cell lines examined. MDAMB-468, BT-20 and MDA-MB-134VI, BT-474 and ZR-75-1 cells expressed the highest levels of caspase-10 mRNA, whereas caspase-10 transcription was significantly lower in ZR-75-30, MDA-MB-483, MDA-MB-330, MDA-MB-361, T47D, MDA-MB-415 and SK-BR-3 cells. Levels of Apaf-1 mRNA were low in MDA-MB-415, SK-BR-3, ZR-75-1, MDA-MB-435s and ZR-75-30 cells. Comparisons of caspase protein and mRNA levels showed some variability, although the data tended to be congruent for each of the caspases.

Relative sensitivity of breast cancer cell lines to TNF- $\alpha$. To determine whether selected caspase expression profiles were associated with responses to different apoptotic stimuli, we first evaluated the sensitivity of selected cell lines to TNF- $\alpha$ induced apoptosis. Each cell line was treated with $80 \mathrm{ng} / \mathrm{ml}$ TNF- $\alpha$ and $5 \mu \mathrm{g} / \mathrm{ml}$ cycloheximide for $4 \mathrm{~h}$, followed by DAPI staining and apoptotic scoring. As shown in Fig. 3, the posttreatment apoptotic index of individual cell lines varied from 18.8 to $58.3 \%$. BT483, HS-578T, T47, MDA-MB-134VI, MDA-MB-231 and MDA-MB415 cells were relatively resistant to TNF- $\alpha$-induced apoptosis, whereas ZR-75-1, ZR75-30, BT-20, MDA-MB-435s, SK-BR-3 and MDA-MB-436 were more sensitive. Since caspase- 8 is a key initiator caspase in apoptosis through the death receptor pathway, we asked whether caspase- 8 expression patterns amongst the cell lines were correlated with $\mathrm{TNF}-\alpha$ responses. In comparison with 


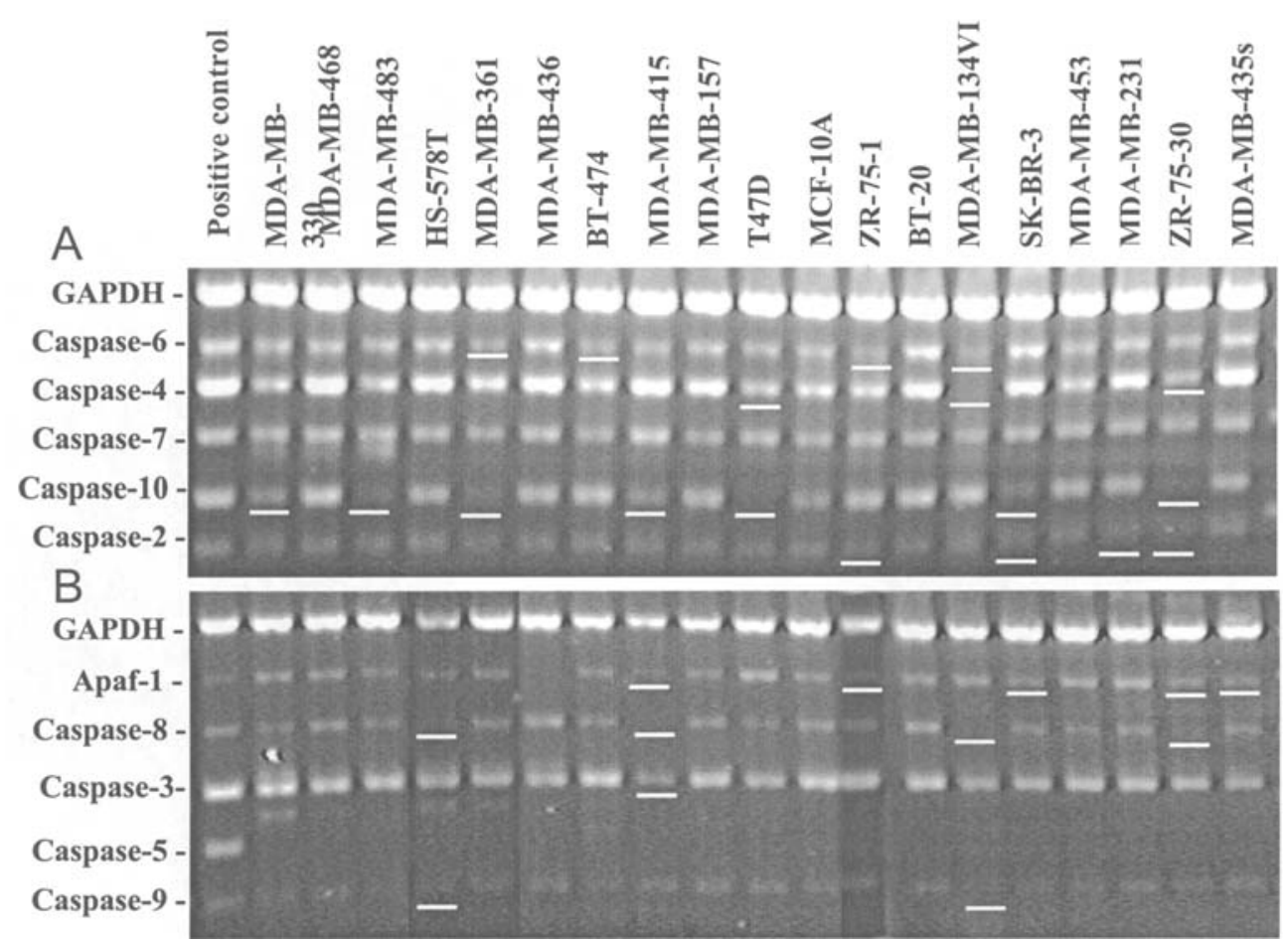

Figure 2. mRNA levels of caspase-2, -4, -6, -7 and 10 (panel A); and caspase- $-3,-5,-8,-9$ and Apaf-1 (panel B) in breast cancer cell lines. Total-RNA was extracted from indicated cell lines. Three micrograms of total-RNA were used for cDNA synthesis. mRNA levels of the selected caspases were amplified by PCR for 30 cycles using a multi-complex PCR kit (set-6) from BioSource according to the manufacturer's protocol. Relative mRNA levels of each caspase were normalized with corresponding GAPDH signals. The caspases with down-regulation of mRNA levels are indicated by a white bar underneath the specific bands.

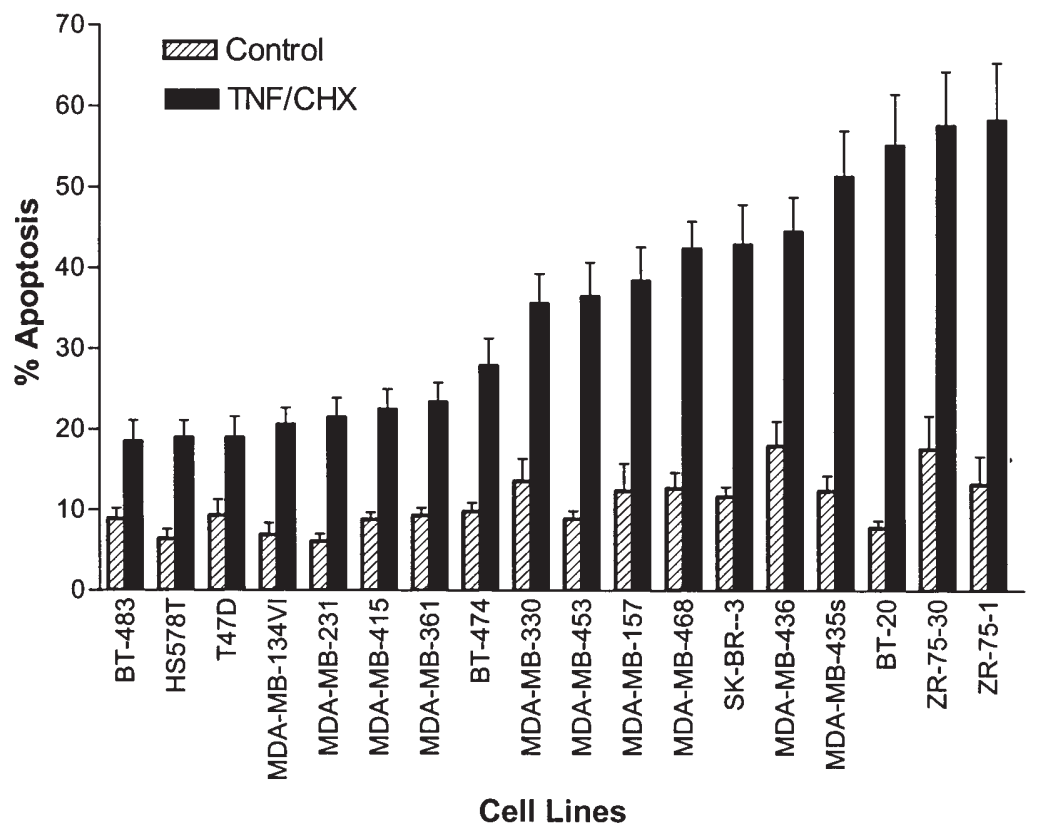

Figure 3. Relative sensitivities of 18 breast cancer cell lines to TNF- $\alpha$-induced apoptosis. Cells from each line were treated with TNF- $\alpha$ plus $5 \mathrm{mg} / \mathrm{ml} \mathrm{CHX} \mathrm{for}$ $4 \mathrm{~h}$. Treated cells were then trypsinized, fixed, stained with DAPI, and attached to a slide using a cytospin. Cells with nuclear condensation and/or fragmentation were counted as apoptotic cells. Apoptotic index (\% apoptosis) was calculated as the percentage of apoptotic cells relative to the total number of cells.

caspase- 8 expression, BT-20, MDA-MB-435S, SK-BR-3 and MDA-MB-436 cells, which were relatively sensitive to TNF- $\alpha$, also expressed higher levels of caspase-8 protein. HS-578T, MDA-MB-483, MDA-MB-415, T47D and MDA-MB-231 cells, which were resistant to TNF- $\alpha$, expressed lower levels of caspase-8. Although there were exceptions (e.g. ZR-75-30 and MDA-MB-361 cells), the apoptotic responses of these breast cancer cell lines to TNF- $\alpha$ were generally associated with caspase-8 levels.

Relative sensitivity of breast cancer cell lines to doxorubicin. The ability of a cancerous cell to undergo apoptosis plays an 


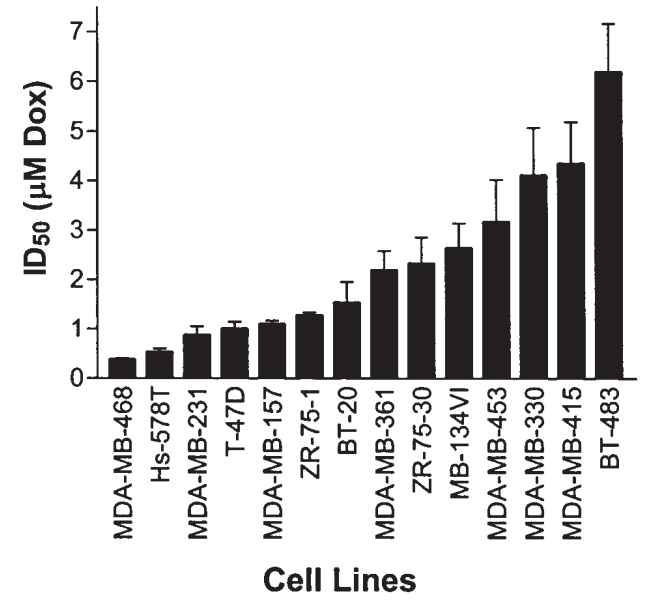

Figure $4 . \mathrm{ID}_{50}$ of breast cancer cell lines in response to doxorubicin (Dox). Breast cancer cell lines listed were treated with Dox at concentrations ranging from 0.15 to $9 \mathrm{mM}$ for 6 days, followed by MTT assay. ID $_{50}$ of each cell line was calculated based on the survival fractions of dose-effect response of Dox-treated cells. The result for each cell line was derived from three separate sets of experiments.

important role in chemo- and radio-therapy response. Because doxorubicin (adriamycin) is commonly used in breast cancer patients, the sensitivity of these selected cell lines to doxorubicin was examined. Fig. 4 shows the $\mathrm{ID}_{50}$ of doxorubicin in 14 breast cancer cell lines. The $\mathrm{ID}_{50}$ of the other 4 cell lines is not shown because their response curves did not fit well with the current model, although these 4 lines appear to be more resistant to doxorubicin. Of the 14 lines shown in Fig. 4, MDA-MB-468, T47D, MDA-MB-231, MDA-MB-157 and BT-20 cells were most susceptible to doxorubicin-induced apoptosis. In general, these cell lines expressed relatively higher mRNA levels of caspase-3, -4, -6, -7 and -10 (with the exception of T47D) and relatively higher levels of caspase- 9 . In contrast, MDA-MB-415, BT-483 and MDA-MB-330 were less sensitive to doxorubicin and expressed lower levels of caspase- 9 and -10 . Hence, the profiles of both apical and effector caspases appeared to be important to doxorubicin sensitivity.

Relative sensitivity of breast cancer cell lines to ionizing radiation. To assess the sensitivity of these same cell lines to ionizing radiation, each cell line was irradiated across a spectrum of doses (from 0.5 to $32 \mathrm{~Gy}$ ) followed by MTT assaybased $\mathrm{ID}_{50}$ determination. As shown in Fig. 5, the responses of 15 breast cancer cell lines (the results from other cell lines were not included because the response curves did not fit well with the $\mathrm{ID}_{50}$ calculation model used) treated with radiation varied significantly. In general, the relative sensitivities of these cell lines were similar to their response to doxorubicin. For example, MDA-MB-468, MDA-MB-231 and MDAMB-157 cells, which were highly sensitive to doxorubicin, were also sensitive to radiation. Similarly, MDA-MB-330 and ZR-75-30 cells, which were resistant to doxorubicin, were also radio-resistant. By correlating caspase expression and mRNA levels of multiple caspases, MDA-MB-134VI cells showed reduced levels of caspase- $6,-4$ and -8 , while mRNA levels of

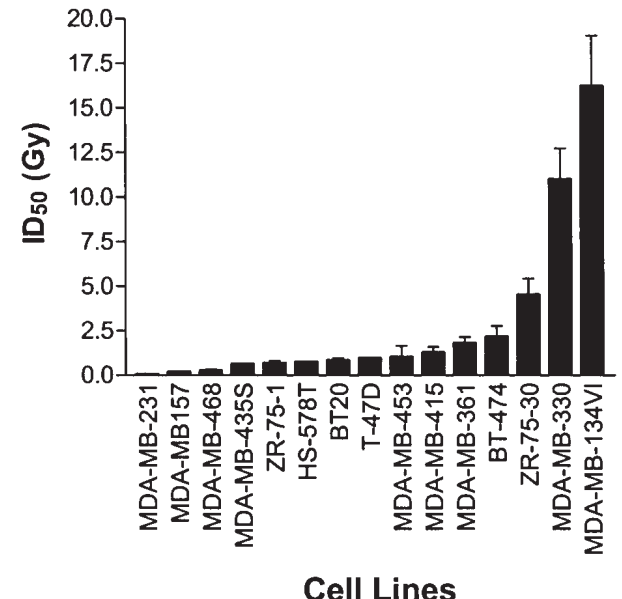

Figure $5 . \mathrm{ID}_{50}$ of breast cancer cell lines in response to radiation. Breast cancer cell lines listed were irradiated at doses ranging from 0.5 to $32 \mathrm{~Gy}$. The survival fractions were detected 6 days post-radiation using MTT assay. $\mathrm{ID}_{50}$ of each cell line was calculated based on the survival fractions of doseeffect response of the irradiated cells. The result for each cell line was derived from three separate sets of experiments.

caspase- 8 and -10 were generally lower in ZR-75-30 cells and mRNA levels of caspase-10 were lower in MDA-MB-330 cells.

\section{Discussion}

We have examined the protein and mRNA expression of multiple caspases in a panel of 18 cell lines as well as the relative sensitivities of these cells to TNF- $\alpha$, doxorubicin and ionizing radiation. We have shown variable caspase expression amongst these lines and our data suggests that caspase expression may be associated with treatment resistance.

We believe that caspase down-regulation and/or deficiency may be a mechanism that contributes to breast carcinogenesis and therapeutic resistance. We and others have previously shown that caspase-3 is deficient in MCF-7 breast cancer cells and that reconstitution of caspase- 3 significantly sensitizes MCF-7 cells to chemotherapy, radiation and granzyme Binduced apoptosis (10-13). Caspase-3 down-regulation/ deficiency has now been detected in $\sim 75 \%$ of breast cancers (14). Our study advances the understanding of caspase expression in breast cancer cells by examining both protein and mRNA of 7 key caspases in 18 breast cancer cell lines. Recent data suggests that caspase alterations may be widely distributed amongst many cancer types. Svingen et al have examined the protein levels of caspase-2, -3, -6, -7, -8, -9 and Apaf-1 expression in the NCI 60-cell-line-panel (only 8 of which are breast cancer cell lines) and they show great variability of caspase expression (17).

Our results identified breast cancer cell lines with low protein levels of certain caspase(s), such as caspase- 2 in MDA-MB-157 and BT 20 cells, caspase-6 in HS-578T cells, caspase-8 in MDA-MB-134VI and ZR-75-30 cells, and caspase-10 in SK-BR-3 and MDA-MB-415 cells. We further demonstrated that variation of the mRNA levels of different caspases was very common among these cell lines. A 
comparison of protein and mRNA results indicates that, in general, the reduced caspase protein levels were associated with lower mRNA levels. For example, both caspase- 8 mRNA and protein levels were down-regulated in MDA-MB-134VI, ZR-75-30, MDA-MB-415 and HS-578T cells. SK-BR-3, T47D and MDA-MB-415 cells, which had lower protein levels of caspase-10, also expressed lower levels of caspase-10 mRNA. However, protein and mRNA levels of some caspases did not behave concomitantly, such as caspase-10 in HS-578T cells and caspase- 8 in MDA-MB157 cells. These results suggest that caspase transcription may be primarily regulated at the transcriptional level but post-transcriptional regulation may also be involved.

In this study we also performed functional screening of selected cell lines by evaluating their apoptotic response to TNF- $\alpha$, doxorubicin and radiation. Although it was a challenge to identify specific caspase profiles with a high correlation to specific treatment responses, we found a general association between caspase- 8 and cellular response to TNF- $\alpha$. Breast cancer cell lines with lower caspase- 8 levels were more resistant to TNF- $\alpha$-induced apoptosis. Given the critical role of caspase- 8 in death receptor-induced apoptosis, these findings might be predicted (as would resistance to other stimuli like Fas ligand and TRAIL).

Results from the treatment studies with doxorubicin and radiation suggest that cell lines that are sensitive to doxorubicin are more likely to be sensitive to radiation as well. This could be because both stimuli induce apoptosis through the DNA damage-mediated apoptotic pathway. Although the correlation between doxorubicin/radiation sensitivity and caspase status was not as apparent as caspase- 8 and TNF- $\alpha$-induced apoptosis, the expression of caspase- 9 and -10 appears to be linked. It is known that caspase- 9 plays an important role in the initiation of apoptosis through the mitochondrial pathway (18). Caspase-10 activation is also involved in DNA damage agentinduced apoptosis (19). Hence, a defect in these caspases may contribute to the resistance of apoptosis through the mitochondrial pathway. We acknowledge that results from these screening assays provide hypothesis-generating information. They suggest that caspase deficiency might predict therapeutic resistance and that caspase reconstitution or upregulation might enhance therapeutic responses.

The observation that down-regulation of caspases is common in breast cancer cell lines raises the question as to how caspase expression may be regulated. Available reports suggest that caspase expression can be modified at both the RNA and the DNA levels. Transcription factors that are involved in the regulation of certain caspases have been identified. For example, AP-4 (activator protein-4) is a transcriptional factor for caspase-9 (20). Transcription of human caspase-7, -8 and -9 is regulated by E2F-1 (21). Hence, an alteration in the activities of these transcription factors and related pathways may cause caspase down-regulation. Caspase deficiency can also be caused by genetic mutations, such as the deletion mutation of the caspase- 3 gene in MCF-3 cells (10). A point mutation of caspase- 8 in A431 cells leads to functional inactivation of caspase-8 (22). Furthermore, down-regulation of caspase- 8 in cancer tissues has been associated with DNA methylation of the caspase- 8 gene (15). Given the significant impact of caspase deficiency/down-regulation in cancer cells and complex mechanisms in the regulation of caspase expression, further study in this direction is likely warranted.

As this project is basically a descriptive study, we will examine caspase expression profiles in more human cancer samples to generate enough data for powerful statistical analysis. Nevertheless, the array style examination of multiple caspases at both protein and mRNA levels and functional screening in these established cell lines have generated information that has many valuable implications. Cell lines bearing significant reductions or increases in caspase expression may be useful in subsequent studies of caspase down-regulation and therapeutic resistance. They may also serve as controls for immunohistochemical examination of caspase expression in cancer tissues. Cell lines with significant down-regulation of caspase mRNA levels can be useful targets to study the role of DNA methylation in the regulation of caspase expression.

\section{Acknowledgements}

This study was supported in part by the Career Development Award DAMD17-99-1-9180 from Department of Defense to X.H.Y. We thank Mr. James Collins for manuscript preparation.

\section{References}

1. Bromberg J: Signal transducers and activators of transcription as regulators of growth, apoptosis and breast development. Breast Cancer Res 2: 86-90, 2000.

2. Barnes DM and Camplejohn RS: P53, apoptosis and breast cancer. J Mammary Gland Biol Neoplasia 1: 163-175, 1996.

3. Krajewski S, Blomqvist C, Franssila K, et al: Reduced expression of proapoptotic gene BAX is associated with poor response rates to combination chemotherapy and shorter survival in women with metastatic breast adenocarcinoma. Cancer Res 55: 4471-4478, 1995 .

4. Tommiska J, Eerola H, Heinonen M, et al: Breast cancer patients with p53 Pro72 homozygous genotype have a poorer survival. Clin Cancer Res 11: 5098-5103, 2005.

5. Reed JC: Mechanisms of apoptosis avoidance in cancer. Curr Opin Oncol 11: 68-75, 1999.

6. Thornberry NA and Lazebnik Y: Caspases: enemies within. Science 281: 1312-1316, 1998.

7. Denault JB and Salvesen GS: Caspases: keys in the ignition of cell death. Chem Rev 102: 4489-4500, 2002.

8. Riedl SJ and Shi Y: Molecular mechanisms of caspase regulation during apoptosis. Nat Rev Mol Cell Biol 5: 897-907, 2004.

9. Fischer U, Janicke RU and Schulze-Osthoff K: Many cuts to ruin: a comprehensive update of caspase substrates. Cell Death Differ 10: 76-100, 2003.

10. Janicke RU, Ng P, Sprengart ML and Porter AG: Caspase-3 is required for alpha-fodrin cleavage but dispensable for cleavage of other death substrates in apoptosis. J Biol Chem 273: 15540-15545, 1998.

11. Yang XH, Sladek TL, Liu X, Butler BR, Froelich CJ and Thor AD: Reconstitution of caspase 3 sensitizes MCF-7 breast cancer cells to doxorubicin- and etoposide-induced apoptosis. Cancer Res 61: 348-354, 2001.

12. Yang XH, Edgerton S and Thor AD: Reconstitution of caspase-3 sensitizes MCF-7 breast cancer cells to radiation therapy. Int $\mathbf{J}$ Oncol 26: 1675-1680, 2005.

13. Yang X, Stennicke HR, Wang B, et al: Granzyme B mimics apical caspases. Description of a unified pathway for transactivation of executioner caspase-3 and -7. J Biol Chem 273: 34278-34283, 1998.

14. Devarajan E, Sahin AA, Chen JS, et al: Down-regulation of caspase 3 in breast cancer: a possible mechanism for chemoresistance. Oncogene 21: 8843-8851, 2002.

15. Hopkins-Donaldson S, Ziegler A, Kurtz S, et al: Silencing of death receptor and caspase- 8 expression in small cell lung carcinoma cell lines and tumors by DNA methylation. Cell Death Differ 10: 356-364, 2003. 
16. Hopkins-Donaldson S, Bodmer JL, Bourloud KB, Brognara CB, Tschopp J and Gross N: Loss of caspase-8 expression in highly malignant human neuroblastoma cells correlates with resistance to tumor necrosis factor-related apoptosis-inducing ligand-induced apoptosis. Cancer Res 60: 4315-4319, 2000.

17. Svingen PA, Loegering D, Rodriquez J, et al: Components of the cell death machine and drug sensitivity of the National Cancer Institute Cell Line Panel. Clin Cancer Res 10: 6807-6820, 2004.

18. Shi Y: Apoptosome: the cellular engine for the activation of caspase-9. Structure 10: 285-288, 2002.

19. Park SJ, Wu CH, Gordon JD, Zhong X, Emami A and Safa AR: Taxol induces caspase-10-dependent apoptosis. J Biol Chem 279: 51057-51067, 2004.
20. Tsujimoto K, Ono T, Sato M, Nishida T, Oguma T and Tadakuma T: Regulation of the expression of caspase- 9 by the transcription factor activator protein-4 in glucocorticoid-induced apoptosis. J Biol Chem 280: 27638-27644, 2005.

21. Nahle Z, Polakoff J, Davuluri RV, et al: Direct coupling of the cell cycle and cell death machinery by E2F. Nat Cell Biol 4: 859-864, 2002.

22. Liu B, Peng D, Lu Y, Jin W and Fan Z: A novel single amino acid deletion caspase- 8 mutant in cancer cells that lost proapoptotic activity. J Biol Chem 277: 30159-30164, 2002. 